\title{
Determinants of Persistent Sputum Smear Positivity after Intensive Phase Chemotherapy among Patients with Tuberculosis at Rhodes Chest Clinic, Nairobi, Kenya
}

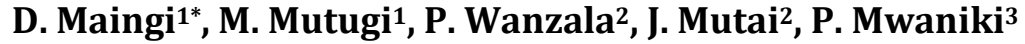 \\ ${ }^{1}$ Jomo Kenyatta University of Agriculture and Technology (JKUAT), Institute of Tropical Medicine and Infectious \\ Diseases, Nairobi, Kenya \\ ${ }^{2}$ Kenya Medical Research Institute (KEMRI), Nairobi, Kenya \\ ${ }^{3}$ School of Health Sciences, Jomo Kenyatta University of Agriculture and Technology (JKUAT), Kenya \\ Email: *wambugudav@gmail.com
}

Received 14 June 2014; revised 29 July 2014; accepted 15 August 2014

Copyright @ 2014 by authors and Scientific Research Publishing Inc.

This work is licensed under the Creative Commons Attribution International License (CC BY). http://creativecommons.org/licenses/by/4.0/

(c) (i) Open Access

\section{Abstract}

The prevalence of TB in sub-Sahara Africa has been reported as 511 per 100,000 populations and a mortality of 74 per 100,000 in year 2009. In the same period, incidence was estimated at 350 cases per 100,000 . In this regard, the health system requires strengthening to respond to the rising cases of infection, drug resistance and quality of life lost while continuing to seek interventions that improve adherence to medication and case detection among those infected. Methods: This study sought to determine factors that are associated with sputum positivity after intensive phase of chemotherapy in people with tuberculosis. It was a retrospective case-control study conducted in Rhodes chest clinic, a City Council health unit in Nairobi that specializes in treatment of chest infections. The participants were sampled from clinic attendants who had completed two months of intensive phase TB chemotherapy and met inclusion criteria. Results: Seventy participants of whom $25(36 \%)$ were sputum positive at the end of two (2) months intensive phase were included in the study. Skipping medication doses was significantly associated with sputum positivity ( $\mathrm{p}=$ 0.01). Patients who were sputum positive at the end of the two-month period were more likely to have taken longer time before seeking treatment compared to those who were sputum negative by median (IQR) $8(3-12)$ and $4(3-8)$ weeks respectively although this difference was not significant $(p=0.09)$. Patients who had not disclosed their infection status had a two-fold possibility of

"Corresponding author.

How to cite this paper: Maingi, D., Mutugi, M., Wanzala, P., Mutai, J. and Mwaniki, P. (2014) Determinants of Persistent Sputum Smear Positivity after Intensive Phase Chemotherapy among Patients with Tuberculosis at Rhodes Chest Clinic, Nairobi, Kenya. Health, 6, 2026-2034. http://dx.doi.org/10.4236/health.2014.615237 
remaining sputum positive at the end of intensive phase. Conclusion: Early diagnosis and treatment of TB, and adherence to medication were important factors that affect sputum conversion during intensive phase of TB treatment. Therefore, public health practitioners should advise patients to seek prompt diagnosis and treatment of signs and symptoms of tuberculosis.

\section{Keywords}

\section{Tuberculosis Treatment in Kenya, Intensive Phase, Persistent Sputum Positivity, TB Drug Resistance, Adherence to TB Medication}

\section{Introduction}

Tuberculosis (TB) is one of the most ancient diseases but remains a substantial public health. Kenya is ranked 13 among 22 high burden countries that collectively contributed about $80 \%$ of world T.B cases [1] [2]. In year 2008, a total of 110,251 cases of T.B were reported to the Division of Leprosy, Tuberculosis and Lung Diseases DLTLD, representing T.B notification rate of 288 per 100,000 population [3]. New cases of TB in year 2007 according to WHO (World Health Organization) were 132,000 thus an incidence rate of 142 cases per 100,000 population.

Although tuberculosis is a curable disease, it is highly infectious and if untreated, each person with active T.B will infect on average 10 to 15 persons in a year [4]. The success of tuberculosis treatment lies in the consistent administration, adherence and completion of treatment. The goal of treatment must therefore be to achieve optimum desirable cure rate. Despite T.B medication in Kenya being free with the hope of achieving high coverage of persons on medication, the cure rate is about $85 \%$ and there is therefore need to examine factors contributing to treatment outcomes so as to improve cure rates and therefore reducing disease burden of morbidity, mortality, relapse, infectiousness, and drug resistance. The first line treatment regimen in Kenya as provided by Division of Leprosy, Tuberculosis and Lung Diseases (DLTLD) entails fixed dose combination (FDC) of Isoniazid (H), Rifampicin (R), Pyrazinamide (Z) and Ethambutol (E) (Table 1).

Some of the factors identified as influencing treatment completion, in a study in Mbagathi Hospital, include, not having enough pills to last until the next available day after a missed appointment date occasioned by delays due to work or family reasons, lack of money for transport and losing pills as some of the main reason to non adherence [5] [6]. The same study, however, observed high treatment adherence to tuberculosis treatment.

This study sought to outline factors that are associated persistent sputum positivity after intensive phase of TB treatment among patients on medication in Rhodes Chest Clinic with the aim of improving clinical outcomes through identification of key factors that promote persistence of sputum positivity and therefore help care providers and policy makers in designing appropriate TB management and monitoring interventions. The findings will also help clinicians optimize sputum conversion and thus improve care outcomes during and after intensive phase of treatment of tuberculosis.

\section{Methods}

\subsection{Study Design}

This was a case control design with cases being patients with positive sputum results after intensive phase of treatment while controls were patients with sputum smears negative results after intensive phase of treatment.

Table 1. 1st line treatment regimen for new adult TB patient.

\begin{tabular}{lll}
\hline Abbreviation of the regimen & & 2RHZE/4RH \\
\hline Phase & Intensive phase & Continuation phase \\
Duration & Directly Observed Treatment (DOT), for two months & $\begin{array}{l}\text { Directly Observed Treatment (DOT), } \\
\text { for four months }\end{array}$ \\
Drugs Used & Rifampicin (R), Isoniazid (H) Pyrazinamide (Z), Ethambutol (E) & Rifampicin (R) and Isoniazid (H),
\end{tabular}




\subsection{Study Area}

This study was conducted at Rhodes Chest Clinic that provides diagnostic, treatment and monitoring for tuberculosis. The health centre (level III) is in Nairobi, the capital city of Kenya with an estimated population of 3 million residents majority of whom are low income earners. It also serves as a referral for several city council of Nairobi clinics for diagnosis, treatment and monitoring of tuberculosis.

\subsection{Study Population}

The study participants were clinic attendants receiving medication and continued TB treatment and care services, they were sampled from patients on tuberculosis treatment willing and consenting to participate in the study. They were required to have completed 2 months intensive phase of TB treatment. A total of 70 persons were sampled of whom 25 patients (cases) were sputum positive after intensive phase of treatment while 45 patients (controls) were sputum negative after intensive phase of treatment.

\subsection{Sample Size and Data Collection}

TB medication compliance has been identified as a significant risk factor for persistent sputum positivity after intensive phase [6]. We postulate that among the cases (sputum positive) $40 \%$ will be non compliant and that among the controls (sputum negative), $10 \%$ will be non compliant. Assuming a power of $80 \%$, alpha of 0.05 and conservative exposure level of 50\% for socio demographic and treatment factors with ratio of 1:2 (case: control), we estimated that we would require a sample size of 72 respondents ( 24 cases and 48 controls). All cases were selected on the basis of their positive sputum microscopy status while controls were selected on condition they had converted to sputum negative after intensive phase. 25 patients on medication for tuberculosis and with positive sputum smear after intensive phase were selected as cases and 45 controls (positive sputum smear after intensive phase) were enlisted in the study. Due to low numbers of the cases, purposive samples were taken while controls were randomly selected. Sputum positive patients were requested to be interviewed even on days that were not their scheduled clinics. The inclusion criteria for cases and controls were: 1) must be an adult (18 years); 2) consenting to taking part in the study; 3) being managed on the recommended 1st line regimen for TB and able to communicate in English or Swahili.

Data collection was through interviewer-administered questionnaires, used to gather information on TB treatment progress while existing patients' records were used to gather information of patients at baseline (at onset of treatment) and sputum results at completion of intensive phase of treatment in addition to clinical outcomes. 3 clinical field assistants, (a clinical officer, a nurse and a records clerk) were recruited and trained on data collection procedures. No pre-scheduled interview appointments were planned and patients presenting as part of routine follow-up visits to the health facilities were requested to participate in the study.

Analysis: Data was entered and analysed using SPSS (16 version). Each questionnaire had a unique identifier to allow validation. Descriptive and inferential statistics were calculated for various social demographic characteristics and treatment outcomes. Univariable logistic regression was used to examine the association between the primary outcome (persistent sputum positivity after intensive phase) and all pre-specified covariates. A multivariable predictive model for persistent sputum positivity after intensive phase was then developed. Gender was included as a priori independent risk factors for persistent sputum positivity with additional variables identified in univariate analysis as associated with persistent sputum positivity $(\mathrm{p}<0.05)$ added into the model starting with those with the strongest association. Variables were retained in the model if a likelihood ratio test (LRT, p value of $<0.05$ ) supported improved model fit. For the univariable and multivariable analyses the odds ratios, accompanying 95\% confidence intervals and Wald test $\mathrm{p}$ values (two-tailed) are reported.

\section{Ethical Considerations}

Scientific and ethical approval for the study were sought and granted by Kenya Medical Research Institute's (KEMRI). Scientific steering committee (SSC) and Ethical Review Committees (ERC) for research to be conducted.

Participation in this study was voluntary. Consent was sought from all study participants with a signed consent form preceding all interviews, minors and those too sick to participate were excluded from the study. There were no benefits or incentives to the participants. Long-term benefit to society is anticipated as results of this survey may be of medical importance. Confidentiality was guaranteed for all information obtained from study 
participants.

\section{Results}

\subsection{Patient Characteristics}

A total of 70 patients who were on TB treatment were recruited into the study at ratio of 1:2 (cases:controls) with 25 of these being cases (persistent sputum positive results after intensive phase). The sex distribution of the respondents was such that males were over three quarters (77\%). In respect to marital status, patients who were married constituted 52\% (37) of the patients while 44\% (31) and 4\% (3) were single and separated respectively.

Patients who were in formal employment (salaried positions) constituted the majority $45 \%$ (32) with those in informal employment being $28 \%$ (20) and the least were the unemployed $27 \%$ (19). Majority of the patients $45 \%$ (32) had an income of $<10000$ while the least $7 \%$ (5) had an income of $>40000$, however $15 \%$ of the patients did not give their income. More descriptive demographics are Table 2.

\subsection{Pre-Medication Factors}

In addition to the socio-demographic factors age, gender, marital status education, occupation and income, the numbers of rooms, no of persons living with per house and duration to seeking treatment were also considered as pre-treatment factors that determined sputum conversion. Of these only duration to seeking treatment was a significant predictor to sputum conversion (Table 2).

Patients who were sputum positive took longer before seeking treatment compared to those who were sputum negative in median weeks (IQR) 8 (3 - 12) and 4 (3 - 8) weeks respectively although not significant (kruskal wallis test $\mathrm{p}=0.09$ ). However patients who took too long before seeking treatment were significantly (kruskal wallis test $\mathrm{p}=0.01$ ) more likely to miss their medication with those who ever missed medication having a median (IQR) 8 (6 - 12) duration to seeking treatment while those who never missed medication had a duration of 4 (3 - 8) weeks (data not shown).

\subsection{Medication Factors}

Majority 89\% of the respondents never forgot to take their medication with only 3\% (2) and 8\% (6) forgetting to take their medication sometimes and rarely respectively. Very few patients were rated as being careless about taking their medication with majority $92 \%$ reporting never being careless. $89 \%$ of the patients were adherent to treatment with less than $1 \%$ reporting either missing or sometimes stopping treatment if they felt better. $30 \%$ (21) of the patients had missed their medication at some point during treatment. Of those who missed treatment, $62 \%$ (13) only missed treatment for one day, 14\% (3) missed treatment for two days while 14\% (3) missed treatment erratically.

Ever missing medication was significantly associated with persistence of sputum positivity (OR 0.20 ; $95 \%$ CI 0.10 - 0.84; $\mathrm{p}=0.023$ ). Although not significant patients who were not offered a HIV test as part of TB care and those who did not disclose their TB status were likely to have an increased odds of sputum positive results by 1.76 (95\% CI 0.11 - 29.38; p = 0.694) times and 2.55 (95\% CI 0.52 - 12.40; p = 0.247) times respectively. Similarly the need for a treatment supporter was not significant (see Table 3).

\subsection{Multivariate Analysis}

In the multivariate analysis, two predictive models for sputum positivity after intensive phase were developed: one for the demographic factors and the other for treatment factors. In Model 1 (Table 4), gender age and income were identified as the main demographic factors predictive of sputum positivity after intensive phase of treatment (LRT 0.053). Regarding treatment factors, gender, age and ever missing medication (LRT 0.05) predicted sputum positivity as shown in Model 2 in Table 5.

\section{Discussion}

The aim of our study was to assess the determinants of persistent sputum smear positivity after intensive phase of chemotherapy. The striking findings from this study were that ever missing drugs was significantly associated with sputum positivity while patients who were sputum positive were likely to have taken longer before seeking 
Table 2. Descriptive and univariate logistic regression analysis for the association of pre-treatment factors between sputum results and covariates.

\begin{tabular}{|c|c|c|c|c|c|c|}
\hline & \multicolumn{2}{|c|}{ Sputum } & \multirow[b]{2}{*}{ Odds Ratio } & \multirow[b]{2}{*}{ Upper CI } & \multirow[b]{2}{*}{ lower CI } & \multirow[b]{2}{*}{ p Value } \\
\hline & Positive & Negative & & & & \\
\hline \multicolumn{7}{|l|}{ Age } \\
\hline Mean (SD) & $37.5(10.8)$ & $35.2(9.6)$ & 1.02 & 0.97 & 1.08 & 0.345 \\
\hline \multicolumn{7}{|l|}{ Gender } \\
\hline male & $22(88)$ & $32(71)$ & 1.00 & & & \\
\hline female & $3(12)$ & 13(29) & 0.32 & 0.08 & 1.26 & 0.103 \\
\hline \multicolumn{7}{|l|}{ Marital } \\
\hline single & $12(48)$ & $19(42)$ & 1.00 & & & \\
\hline married & $11(44)$ & $25(56)$ & 0.76 & 0.28 & 2.06 & 0.59 \\
\hline separated & 2(8) & $1(2)$ & 3.17 & 0.26 & 38.84 & 0.367 \\
\hline \multicolumn{7}{|l|}{ Religion } \\
\hline Christian & $24(96)$ & $44(98)$ & 1.00 & & & \\
\hline others & $1(4)$ & $1(2)$ & 1.76 & 0.11 & 29.38 & 0.694 \\
\hline \multicolumn{7}{|l|}{ Education } \\
\hline primary & $10(40)$ & $14(31)$ & 1.00 & & & \\
\hline secondary & $11(44)$ & $18(40)$ & 0.78 & 0.26 & 2.31 & 0.651 \\
\hline tertiary & $3(12)$ & $9(20)$ & 0.42 & 0.09 & 1.95 & 0.271 \\
\hline university & $1(4)$ & $4(9)$ & 0.32 & 0.03 & 3.27 & 0.335 \\
\hline \multicolumn{7}{|l|}{ Occupation } \\
\hline unemployed & $7(28)$ & $12(27)$ & 1.00 & & & \\
\hline informal & $13(52)$ & $18(40)$ & 1.33 & 0.42 & 4.27 & 0.628 \\
\hline formal & $5(20)$ & $15(33)$ & 0.57 & 0.14 & 2.26 & 0.425 \\
\hline \multicolumn{7}{|l|}{ Income } \\
\hline$<10000$ & $14(56)$ & $17(38)$ & 1.00 & & & \\
\hline $10000-20000$ & $4(16)$ & $12(27)$ & 0.38 & 0.10 & 1.42 & 0.151 \\
\hline $20000-30000$ & $1(4)$ & $6(13)$ & 0.19 & 0.02 & 1.75 & 0.143 \\
\hline$\geq 40000$ & $1(4)$ & $4(9)$ & 0.28 & 0.03 & 2.82 & 0.282 \\
\hline \multicolumn{7}{|l|}{ No of rooms } \\
\hline Median (IQR) & $1(1-2)$ & $1(1-2)$ & 0.80 & 0.42 & 1.53 & 0.496 \\
\hline \multicolumn{7}{|l|}{ No of persons per house } \\
\hline Median (IQR) & $2(1-4)$ & $2(1-4)$ & 0.90 & 0.71 & 1.16 & 0.43 \\
\hline \multicolumn{7}{|l|}{ Duration to treatment (weeks) } \\
\hline Median (IQR) & $8(3-12)$ & $4(3-8)$ & 1.09 & 0.98 & 1.20 & 0.101 \\
\hline
\end{tabular}


Table 3. Descriptive and univariate logistic regression analysis for the association of treatment related factors between sputum results and covariates.

\begin{tabular}{|c|c|c|c|c|c|c|}
\hline \multicolumn{7}{|c|}{ Sputum } \\
\hline & Positive & Negative & Odds Ratio & Upper CI & Lower CI & p Value \\
\hline \multicolumn{7}{|c|}{ Ever missed medication } \\
\hline yes & $12(48)$ & $9(20)$ & 1.00 & & & \\
\hline no & $13(52)$ & $36(80)$ & 0.29 & 0.10 & 0.84 & 0.023 \\
\hline \multicolumn{7}{|c|}{ Reason missed medication } \\
\hline side effect & $3(12)$ & $2(4)$ & 1.00 & & & \\
\hline forgot & $7(28)$ & $4(9)$ & 1.17 & 0.13 & 10.22 & 0.889 \\
\hline \multicolumn{7}{|c|}{ Require a treatment supporter } \\
\hline yes & $18(72)$ & $32(71)$ & 1.00 & & & \\
\hline no & $3(12)$ & $8(18)$ & 0.89 & 0.23 & 3.37 & 0.862 \\
\hline Don’t know & $4(16)$ & $5(11)$ & 1.42 & 0.34 & 5.98 & 0.631 \\
\hline \multicolumn{7}{|c|}{ Requested HIV test as TB care } \\
\hline yes & 24(96) & 44(98) & 1.00 & & & \\
\hline no & $1(4)$ & $1(2)$ & 1.76 & 0.11 & 29.38 & 0.694 \\
\hline \multicolumn{7}{|c|}{ Ever disclose TB status } \\
\hline yes & $21(84)$ & $42(93)$ & 1.00 & & & \\
\hline no & $4(16)$ & $3(7)$ & 2.55 & 0.52 & 12.40 & 0.247 \\
\hline
\end{tabular}

Table 4. Multivariate model for demographic factors that predict sputum positivity.

\begin{tabular}{|c|c|c|c|c|c|c|}
\hline & & Odds Ratio & Upper CI & Lower CI & p Value & LRT \\
\hline \multirow[t]{3}{*}{ Gender } & & & & & & 0.053 \\
\hline & Male & 1.00 & & & & \\
\hline & Female & 0.14 & 0.01 & 1.29 & 0.083 & \\
\hline \multirow{6}{*}{$\begin{array}{l}\text { Age in years } \\
\text { Income }\end{array}$} & & 1.04 & 0.97 & 1.11 & 0.271 & \\
\hline & & & & & & \\
\hline & $<10,000$ & 1.00 & & & & \\
\hline & $10,000-20,000$ & 0.19 & 0.04 & 0.86 & 0.032 & \\
\hline & $20,000-30,000$ & 0.16 & 0.02 & 1.61 & 0.119 & \\
\hline & $<40,000$ & 0.18 & 0.01 & 2.18 & 0.176 & \\
\hline
\end{tabular}

Table 5. Multivariate model for Treatment factors that predict sputum positivity.

\begin{tabular}{|c|c|c|c|c|c|c|}
\hline & & Odds Ratio & Upper CI & Lower CI & p Value & LRT \\
\hline \multirow[t]{3}{*}{ Gender } & & & & & & 0.05 \\
\hline & Male & (base) & & & & \\
\hline & Female & 0.20 & 0.02 & 1.81 & 0.152 & \\
\hline \multirow{4}{*}{$\begin{array}{l}\text { Age in years } \\
\text { missed medica }\end{array}$} & & 1.03 & 0.97 & 1.09 & 0.357 & \\
\hline & & & & & & \\
\hline & Yes & (base) & & & & \\
\hline & No & 0.30 & 0.09 & 1.06 & 0.061 & \\
\hline
\end{tabular}


treatment when compared to those who were sputum negative which is consistent with findings from other studies.

\subsection{Pre-Treatment Factors Affecting Sputum Conversion}

Considerations of patient's socio-demographic and treatment-related factors have been found to be significant determinants of tuberculosis treatment outcome. In this study, there is a suggestive trend for a decrease in the risk of sputum positivity with increase in education level although this association was not significant. However, these results compare with those from Panambuco, Brazil by Magda and colleagues [7] which highlighted that complete or incomplete secondary or university education was associated with a lower risk of defaulting from TB treatment. It is believed that a low level of education hinders perception of the seriousness of the disease and causes difficulties in understanding medical guidelines. Further, the more one is educated the higher the chance of conceptualizing the danger of non adherence and the importance of treatment compliance.

Although TB medication is free, other indirect costs associated with TB infection like lost income, transport, food costs due to the extra nutritional requirements among others may hinder treatment uptake and adherence. This could therefore explain the decreased risk of sputum positivity associated with medication adherence among individuals in formal employment and those having an income of greater than ksh 10,000 (ksh $85=1$ USD).

The decrease in the risk of being sputum positive after intensive phase for every increase in one room in the house may allude to the assumption that with congestion, there are chances of re-infection with the mycobacterium if the patient is living in enclosed environment where they may have infected other people living with them.

\subsection{Treatment Factors}

\subsubsection{HIV Testing and TB Status Disclosure}

Not disclosing TB status had a two fold increase in risk of sputum positivity while not being offered HIV services alongside TB treatment increased risk of sputum positivity. Which highlight the possibility of reinforcing messages that are given during counseling on importance of treatment and adherence which are common in both HIV and TB. This finding highlights the fears that people have regarding disclosure of the illnesses and hence the need for disclose to a suitable person who could provide the role of a treatment supporter actively or passively. It also shows that stigma and discrimination may be related to tuberculosis.

\subsubsection{Adherence to Medication}

A great challenge to a tuberculosis control programme is ensuring that TB patients seek diagnosis in a timely manner and, once diagnosed, adhere to treatment. Central to this inquiry was the assessment of adherence to treatment medication using Morisky adherence scale [8]. Missing drugs was significantly associated with sputum positivity. Those who poorly complied with medication had an increased risk of sputum positivity i.e. rarely/sometimes careless about drugs, forgot or even stopped medication. These finding may be explained on the basis of low or suboptimal levels to suppress multiplication of the mycobacterium tuberculosis. Several studies have been undertaken on the relationship between adherence to medication and persistent sputum positivity [9]-[11].

\subsubsection{Time to Diagnosis and Initiation of Treatment}

Time from onset of symptoms to diagnosis and initiation of tuberculosis chemotherapy has in this study been found to be significant to persistence in sputum positivity after intensive phase. The longer one takes to have a diagnosis and initiate treatment, the higher the chance of sputum positivity after completion of intensive phase. A study in Gambia reported the median delay from onset of symptoms to commencement of treatment was 8 weeks (range 4 -12) in the urban area which compares with the similar median of 8 weeks and IQR of 3 to 12 week reported in this study [12]. The study in Gambia however did not establish a relationship between duration of delay to treatment and cure rate, but longer delay did increase the risk of death [12]. Banu and colleagues in India [13] indicated that pulmonary cavitation is associated with persistent sputum positivity after intensive phase chemotherapy. Though this study did not seek to establish the extent of the disease by the time of initiation of medication, it is possible that delayed diagnosis and treatment initiation leads to adverse effect to the 
lung tissue.

These findings are consistent with those from another recent study in South Africa by Calver et al. [10] which showed that diagnosis delay and inappropriate therapy facilitate disease transmission and drug-resistance. This finding therefore calls for implementation of rapid diagnostics and enhanced active screening strategies, for all with coughs for other signs and symptoms of tuberculosis. All persons with unexplained weight loss, unexplained persistent cough for more than 2 weeks, and unexplained night sweats should be investigated for tuberculosis.

\subsubsection{Time to Diagnosis and Missing Medication}

In this study, it was further established that patients who took long (median time of 8 weeks) to seek diagnosis and treatment had higher likelihood of missing medication during treatment. It is possible that the delay in seeking treatment may be characterized by denial that may spill over to the treatment phase. This calls for adherence counseling and for close monitoring of this category of patients by health care providers.

\subsubsection{Limitations of the Study}

Despite these positive findings these results should be interpreted in the light of the following drawbacks. Firstly, we did not take into account results of culture and there is a possibility that some of the bacilli detected in the sputum were dead, however the action on whether to institute additional period for intensive phase are based on sputum microscopy findings in developing world. Secondly, we did not attempt to have sputum samples reread to validate the findings, however the laboratory in which these investigations were carried out are accredited. Other general limitations are the draw backs associated with this type of study such as recall bias. Despite the above mentioned limitations the following strengths reinforce our arguments. There was a deliberate effort to ensure construct validity at design level of the questionnaire and further external validity of the study finding.

\section{Conclusion}

Treatment adherence to TB medication is associated with desirable treatment outcome while sub-optimal cure rates pose a risk in the increase in the multi drug resistant strains which have recently been reported to be on the rise in Kenya. Therefore to guard against expensive to treat form of drug resistant TB, success with first line treatment must be optimized. An early reliable indicator of treatment outcome is sputum smear status. Further, more research is required to explore how adherence to treatment and time to diagnosis overly affects TB treatment outcomes.

\section{Acknowledgements}

The authors are grateful to the staff of Rhodes chest clinic and the patients who consented to participate in the study.

\section{Contributions}

The idea for the study and its design were conceived David Maingi (DM), with advice from Mutugi Marion (MM), Wanzala Peter (WP), Mutai James (MJ), Mwaniki Peter (MP). DM was responsible for data collection, and data entry. DM, MM, WP PM MJ MP were responsible for data analyses and DM prepared the initial draft manuscript. All authors reviewed the draft manuscript and provided input to and approval for the final version of the report.

\section{Conflict of Interest Statement}

There are no conflicts of interest.

\section{References}

[1] Maher, D.R.M. (2005) Global Epidemiology of Tuberculosis. Clinics in Chest Medicine, 26, 167-182.

[2] Saito, M.B.C., Gilman, R.H., Bowering, A., Levy, M.Z. and Evans, C.A. (2004) The Value of Counting BCG Scars for 
Interpretation of Tuberculin Skin Tests in a Tuberculosis Hyperendemic Shantytown, Peru. The International Journal of Tuberculosis and Lung Disease, 14, 429-437.

[3] Division of Leprosy, Tuberculosis and Lung Diseases, Ministry of Public Health and Sanitation (2009) Guidelines for TB and Leprosy Control. Nairobi, Kenya.

[4] WHO Stop TB Partnership (2006) The Global Plan to Stop TB, 2006-2015. Actions for Life: Towards a World Free of Tuberculosis. 10, 240-1.

[5] Githui, W.A., et al. (2004) Isolation of Multidrug-Resistant Tuberculosis Strains in Patients from Private and Public Health Care Facilities in Nairobi, Kenya. The International Journal of Tuberculosis and Lung Disease, 8, 837-841.

[6] Raguenad, M.Z.R., Massaquoi, M., Ombeka, V., Ritter, H. and Chakaya, J.M. (2008) High Adherence to Anti-Tuberculosis Treatment among Patients Attending a Hospital and Slum Health Centre in Nairobi, Kenya. Global Public Health: An International Journal for Research, Policy and Practice, 3, 433-439.

[7] Maruza, M., et al. (2011) Risk Factors for Default from Tuberculosis Treatment in HIV-Infected Individuals in the State of Pernambuco, Brazil: A Prospective Cohort Study. BMC Infectious Diseases, 11, 351.

[8] Morisky, D.E., et al. (2008) Predictive Validity of a Medication Adherence Measure in an Outpatient Setting. Journal of Clinical Hypertension (Greenwich, Conn.), 10, 348-354.

[9] Munro, S.A., Lewin, S.A., Smith, H.J., Engel, M.E., Fretheim, A., et al. (2007) Patient Adherence to Tuberculosis Treatment: A Systematic Review of Qualitative Research. PLoS Med, 4.

[10] Calver, A.D., et al. (2010) Emergence of Increased Resistance and Extensively Drug-Resistant Tuberculosis despite Treatment Adherence, South Africa. Emerging Infectious Disease, 16, 264-271.

[11] Chang, K.C., et al. (2004) Risk Factors for Defaulting from Anti-Tuberculosis Treatment under Directly Observed Treatment in Hong Kong. The International Journal of Tuberculosis and Lung Disease, 8, 1492-1498.

[12] Lienhardt, C., et al. (2001) Factors Affecting Time Delay to Treatment in a Tuberculosis Control Programme in a SubSaharan African Country: The Experience of the Gambia. The International Journal of Tuberculosis and Lung Disease, 5, 233-239.

[13] Banu Rekha, V.V., et al. (2007) Sputum Conversion at the End of Intensive Phase of Category-1 Regimen in the Treatment of Pulmonary Tuberculosis Patients with Diabetes Mellitus or HIV Infection: An Analysis of Risk Factors. Indian Journal of Medical Research, 126, 452-458. 
Scientific Research Publishing (SCIRP) is one of the largest Open Access journal publishers. It is currently publishing more than 200 open access, online, peer-reviewed journals covering a wide range of academic disciplines. SCIRP serves the worldwide academic communities and contributes to the progress and application of science with its publication.

Other selected journals from SCIRP are listed as below. Submit your manuscript to us via either submit@scirp.org or Online Submission Portal.
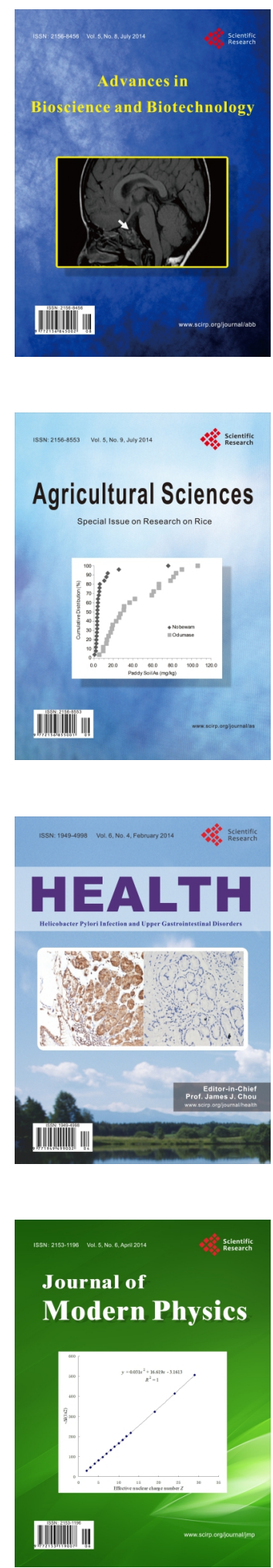
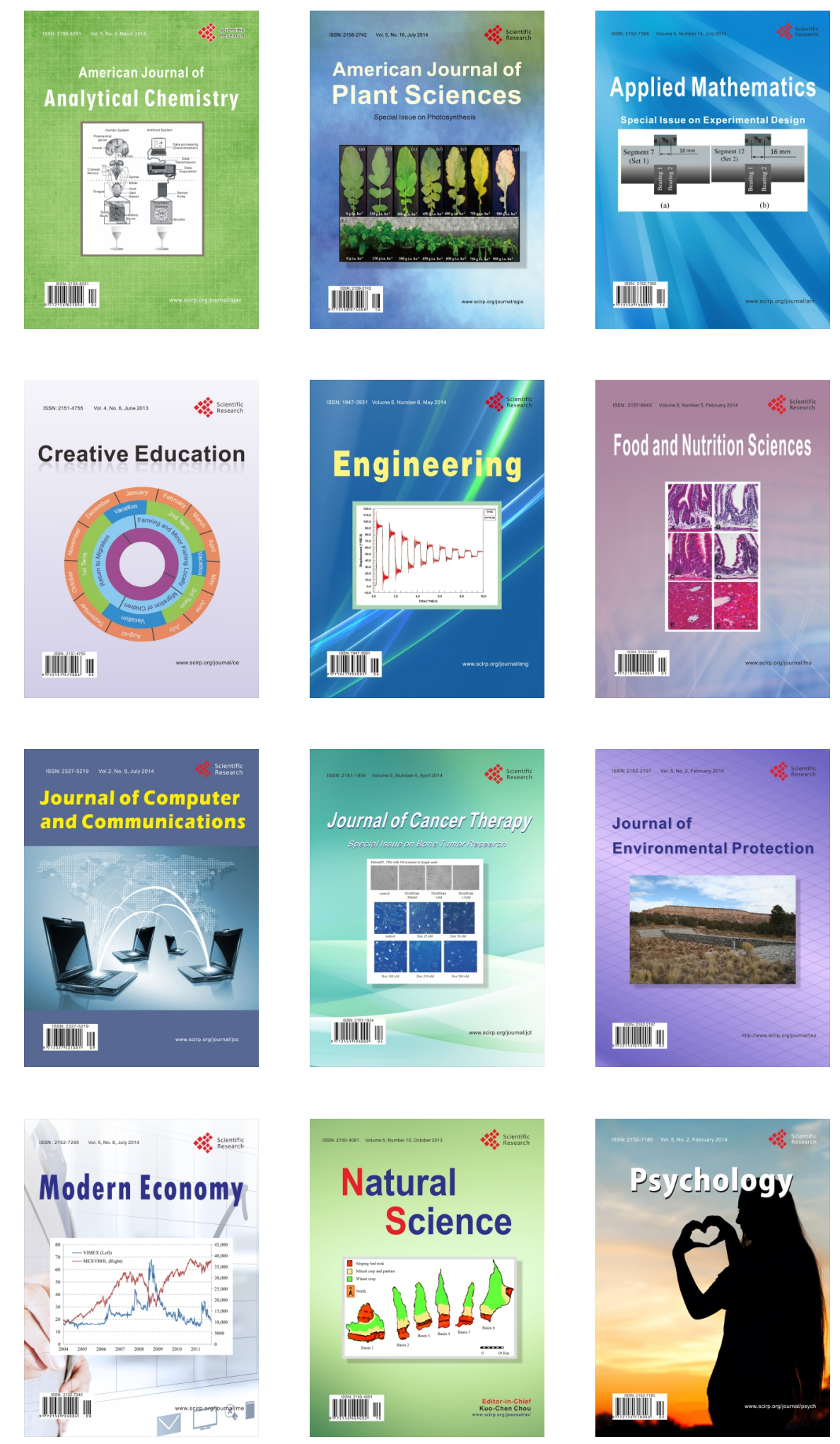